\title{
Treatment of asthma by the inhaled corticosteroid ciclesonide given either in the morning or evening
}

\author{
D.S. Postma*, C. Sevette ${ }^{\#}$, Y. Martinat ${ }^{\mp}$, N. Schlösser ${ }^{+}$, J. Aumann ${ }^{\S}$, H. Kaféf
}

Treatment of asthma by the inhaled corticosteroid ciclesonide given either in the morning or evening. D.S. Postma, C. Sevette, Y. Martinat, N. Schlösser, J. Aumann, H. Kafé. C ERS Journals Ltd 2001.

ABSTRACT: The study addressed the question whether the novel inhaled prodrug corticosteroid ciclesonide is equally effective when inhaled in the morning compared to the evening.

For this purpose a double-blind, randomized, parallel group study was initiated in which 209 asthmatic patients (forced expiratory volume in one second $=50-90 \%$ predicted) inhaled either $200 \mu \mathrm{g}$ ciclesonide in the morning or in the evening, for 8 weeks. Efficacy was assessed by means of spirometry as well as daily recordings of morning and evening peak expiratory flow (PEF), symptoms and use of rescue medication. The 24-h urinary cortisol excretion was measured to evaluate any effect on hypothalamic-pituitary-adrenol axis.

Ciclesonide significantly improved asthma control. Morning and evening administration was shown to be equally effective for the different spirometry variables, evening PEF, symptoms, use of rescue medication and number of asthma exacerbations. Regarding morning PEF, the improvements after evening dosing were more prominent and equivalence of morning and evening administration could not be demonstrated. No relevant influence on cortisol excretion was found.

Overall, the study indicates that ciclesonide can be given either in the morning or in the evening to meet the patients' preference and individual medical needs, although evening administration may lead to a more pronounced improvement in morning peak expiratory flow.

Eur Respir J 2001; 17: 1083-1088.

Inhaled corticosteroids have become the mainstay of therapy for patients with asthma. However, as with other inhaled or oral asthma drugs, compliance to inhaled steroids is often poor [1]. Among the manifold reasons underlying noncompliance, complicated regimens and dosing frequency are considered to be significant factors. Initially, inhaled steroids were recommended to be used four-times a day. Although such a regimen might be more effective than twice-daily dosing [2], in daily life higher compliance with a twicedaily regimen might well compensate reduced efficacy [3]. Meanwhile numerous studies have shown that in the majority of patients a twice-daily schedule can effectively control asthma and this is presently the standard scheme. Additionally, it was suggested that decreasing the dosing frequency to once daily might further enhance adherence to the prescribed regimen [4]. In the past the efficacy of once-daily administration was, therefore, tested with a number of inhaled steroids in patients with mild-to-moderate asthma [5].

Ciclesonide is a novel prodrug glucocorticosteroid in development for the treatment of asthma. Ciclesonide which has a chiral centre in the acetal side chain, exists as two epimers with different receptor affinities and metabolization rates. Only R-ciclesonide was selected for clinical development (referred to as ciclesonide from now on). Ciclesonide itself is inactive and needs to be cleaved by esterases to bind to the
*Dept of Pulmonology, University Hospital, Groningen, the Netherlands, ${ }^{\#}$ Centre Hospitalier, Perpignan, France, Centre Médical Parot, Lyon, France, ${ }^{+}$Central Military Hospital, Heidelberglaan, Utrecht, the Netherlands, ${ }^{9}$ Kunstlaan, Hasselt, Belgium and ${ }^{f}$ Centre de Pneumologie, Saint-Quentin, France.

Correspondence: D.S. Postma, Dept of Pulmonology, University Hospital, Hanzeplein 1, NL-9713 Groningen, The Netherlands.

Fax: 31503619320

Keywords: Asthma

dosing time

inhaled steroids

once daily

Received: November 222000

Accepted after revision March 92001

The study was supported by Byk Gulden Pharmaceuticals. glucocorticoid receptor. The efficacy of ciclesonide in humans was demonstrated in two placebo-controlled trials showing a dose-dependent reduction of airway hyperresponsiveness to adenosine-5'-monophosphate [6] and a significant inhibition of early and late phase reaction after allergen challenge [7].

Ciclesonide is currently developed for once-daily dosing in patients with mild-to-moderate asthma. In the majority of clinical trials carried out so far it was administered as a single dose in the morning and a daily dose of $200 \mu \mathrm{g}$ was shown to be superior to placebo. In order to tailor asthma management of individuals, a flexible dosing time would be ideal.

The present study, therefore, addressed the question whether the time point of administration (either morning or evening) affects the efficacy of ciclesonide. Based on the results for budesonide [8] the hypothesis was put forward that morning and evening dosing of ciclesonide are equi-effective.

\section{Patients and methods}

\section{Patients}

Outpatients of either sex, aged $18-75$ yrs, with a history of bronchial asthma as defined by American Thoracic Society (ATS) criteria [9] were included. 
Patients were eligible to enter the baseline period if they had used rescue medication only during the past 4 weeks and their forced expiratory volume in one second (FEV1) ranged 50-90\% predicted. Patients being pretreated with inhaled steroids (daily doses of up to $500 \mu \mathrm{g}$ beclomethasone dipropionate (BDP) or flunisolide, $400 \mu \mathrm{g}$ budesonide and $250 \mu \mathrm{g}$ fluticasone propionate for at least 4 weeks) were also eligible if their FEV1 was $80-100 \%$ pred. The inhaled steroids were withdrawn at the start of the baseline period. For randomization, FEV1 had to be $50-90 \%$ pred in all patients. In addition, the patients had to show reversibility $(\triangle \mathrm{FEV} 1 \geqslant 15 \%$ initial) after inhalation of $200-400 \mu \mathrm{g}$ salbutamol either during the baseline period or the past 3 months.

Patients were excluded if they had either an asthma exacerbation, an infection of the lower airways, a hospital admission for asthma or if they used systemic steroids in the 4 weeks prior to start of baseline. Patients were also excluded if they suffered from chronic obstructive pulmonary disease (COPD) and/or other relevant lung diseases, or were heavy (ex-) smokers with $\geqslant 10$ pack-yrs. Pregnant, lactating and premenopausal females without safe contraception were ineligible.

Written informed consent was obtained from each patient prior to entering the trial. The protocol was approved by the Ethics Committees of the individual investigators.

\section{Study protocol}

The study had a double-blind, randomized, parallel group design. Following a baseline period of $1-4$ weeks, the patients were randomly allocated to one of two treatment groups: either ciclesonide in the morning (treatment group "morning") or ciclesonide in the evening (treatment group "evening"). Each patient inhaled one puff of $200 \mu \mathrm{g}$ ciclesonide at the time point indicated on the label of the inhaler and one puff of placebo at the alternate time point for 8 weeks. Ciclesonide was administered by metered-dose inhaler (MDI) using 1, 1, 1, 2-hydrofluoralkane (HFA) $134 \mathrm{a}$ as propellant.

The patients visited the investigational sites at weekly intervals during the baseline period and at 4-weekly intervals during the treatment period or whenever their asthma deteriorated. At each visit lung function was measured and adverse events were elicited by open questioning. At the start of the baseline period, as well as at the end of the treatment period (or upon patient withdrawal), a physical examination including 12-lead electrocardiography (ECG) and a standard safety laboratory work-up was performed. In order to assess the effect of ciclesonide on hypothalamic-pituitary-adrenal (HPA) axis patients collected their 24-h urine at home after 1 week of baseline, as well as at the end of the treatment period and cortisol excretion was determined. Creatinine was also measured in urine to allow for correction in case random or limited time collections were made. The cortisol radioimmunoassay used did not interfere with ciclesonide or its active metabolite.
In an individual subject, all lung function readings (FEV1, forced vital capacity (FVC)) had to be performed within $\pm 1.5 \mathrm{~h}$ referenced to the randomization visit. The rescue medication had to be withheld for $\geqslant 4 \mathrm{~h}$ prior to each measurement. The highest value from at least three technically satisfactory attempts was used for analysis. Predicted values were calculated according to the formula of the European Coal and Steel Community [10].

No other asthma drugs except rescue medication and trial medication were allowed throughout the trial. Short-acting $\beta$-agonists (administered by MDI or powder inhaler) were used to relieve symptoms and the same rescue medication (drug and device) was to be utilized throughout the trial. Cromones (as nasal spray or eyedrops) and histamine 1 receptor (H1)blockers were allowed to treat symptoms of allergic rhinitis. Nasal and dermatological steroids were not permitted during the 4 weeks before the start of the baseline period as well as during the study. In case treatment with oral steroids became necessary because of an asthma exacerbation, the patient had to be withdrawn from the trial. Those cases were handled as "lack of efficacy" and included in the per-protocol end-point analysis.

Throughout the trial the patients recorded peak expiratory flow (PEF) (Roland $\AA$ Pulmo-Test AS, Roland Arzneimittel, Hamburg, Germany) daily in the morning, immediately after getting up, and in the evening between 16:00-20:00 h. At the same time symptoms as noted during the night and day, respectively, were recorded applying a four-point scale (i.e. the maximum daily score was 8). Additionally, the daily use of the rescue medication had to be documented in a diary. For the diary variables, the mean of all entries made during the week prior to a visit was used for analysis. For the sake of brevity, the average of a weeks measurements of morning PEF are referred to as "morning PEF". The same holds true for accounts of all other diary card variables. Both the investigator and the patient assessed the effectiveness of the trial medication according to a four-point scale: very effective (good control of asthma), effective (not optimal, but acceptable asthma control), slightly effective (moderate asthma control, improvement desired), ineffective (poor control of asthma).

\section{Statistical analysis}

Primary efficacy variable was the weekly average of morning PEF (average of last week of study versus average of last week of baseline). A sample size of 86 patients per group gives $90 \%$ power to correctly conclude equivalence in morning PEF in case of no treatment difference, $\alpha=0.05$, two-sided, equivalence range of $\pm 25 \mathrm{~L} \cdot \mathrm{min}^{-1}$ for the difference in pre/post changes, for which an SD of $50 \mathrm{~L} \cdot \mathrm{min}^{-1}$ was assumed [11]. Within and between treatment comparisons were based on the differences between end and start of treatment. For lung function variables, equivalence of morning versus evening administration was assessed by analysis of covariance with baseline value and age as covariables, and sex and centre as factors. 
Table 1. - Patient characteristics

\begin{tabular}{|c|c|c|c|c|}
\hline \multirow[t]{2}{*}{ Variable } & \multicolumn{2}{|c|}{ Itt analysis } & \multicolumn{2}{|c|}{ pp analysis } \\
\hline & Morning & Evening & Morning & Evening \\
\hline Patients $n$ & 110 & 99 & 88 & 80 \\
\hline $\mathrm{M}: \mathrm{F}$ & $65: 45$ & $49: 50$ & $54: 34$ & $40: 40$ \\
\hline Age yrs* & $39(19-75)$ & $38(18-68)$ & $39(19-75)$ & $36(18-68)$ \\
\hline Nonsmokers:(Ex-)Smokers & $61: 49$ & $54: 45$ & 49:39 & $43: 37$ \\
\hline Pretreated:nonpretreated with ICS & $41: 69$ & $37: 62$ & $31: 57$ & $28: 52$ \\
\hline \multicolumn{5}{|l|}{ ICS pretreatment dose in } \\
\hline BDP equivalents $\mu \mathrm{g}^{*}$ & $500(50-1000)$ & $500(200-500)$ & $500(200-500)$ & $500(200-500)$ \\
\hline FEV1 \% predicted & $77 \pm 9$ & $77 \pm 11$ & $77 \pm 9$ & $75 \pm 11$ \\
\hline Reversibility $\%$ baseline & $23.5 \pm 11$ & $22.8 \pm 11$ & $24.5 \pm 11$ & $23.8 \pm 12$ \\
\hline Morning PEF \% predicted & $90 \pm 18$ & $87 \pm 20$ & $90 \pm 20$ & $86 \pm 8$ \\
\hline
\end{tabular}

Equivalence acceptance limits for FEV1 and FVC changes were chosen as $200 \mathrm{~mL}$. In this equivalence trial, the per-protocol analysis was considered as primary analysis and the intention-to-treat analysis as a secondary one [12]. Treatment differences in the number of drop-outs due to lack of efficacy were analysed by Fisher's exact test. Changes in symptom scores and use of rescue medication were analysed nonparametrically (Wilcoxon-Pratt signed rank test within groups, Mann-Whitney U-Test between groups). Generally, two-sided $95 \%$ confidence limits were given for treatment differences. Data are presented as mean \pm SD unless stated otherwise.

\section{Results}

Out of 270 patients enrolled into the baseline period, 209 were randomized. The protocol was violated by 41 patients and these were excluded from the per-protocol analysis which finally comprised 88 patients in the morning group and 80 patients in the evening group (tables 1 and 2 for baseline characteristics). As the study aimed for equivalence, the efficacy results for the perprotocol population are being reported; the results from

Table 2.-Baseline effectiveness variables for patients in the per-protocol analysis

\begin{tabular}{|c|c|c|}
\hline Variable & Morning & Evening \\
\hline EV1 L & $2.72 \pm 0.67$ & 0.71 \\
\hline FVC L & $3.84 \pm 1.01$ & \\
\hline Morning PEF $\mathrm{L} \cdot \mathrm{min}^{-1}$ & $446 \pm 131$ & $424 \pm 117$ \\
\hline Evening PEF $\mathrm{L} \cdot \mathrm{min}^{-1}$ & $465 \pm 123$ & $440 \pm 122$ \\
\hline 1 daily symptom score* & $1.57(0-5.26)$ & $1.57(0-7.0)$ \\
\hline $\begin{array}{l}\text { Daily use rescue medication, } \\
\text { puffs } 24 \mathrm{~h}^{-1 *}\end{array}$ & $1.29(0-9.0)$ & $1.76(0-16.83)$ \\
\hline \multicolumn{3}{|c|}{$\begin{array}{l}\text { *: Data are presented as median (range). For the diary } \\
\text { parameters (morning and evening peak expiratory flow } \\
\text { (PEF) symptoms, use of rescue medication) the average over } \\
\text { the last } 7 \text { days prior to start of treatment is being reported. } \\
\text { FEV1: forced expiratory volume in one second; FVC: forced } \\
\text { vital capacity. }\end{array}$} \\
\hline
\end{tabular}

the intention-to-treat analysis are, however, well in agreement with the per-protocol analysis. With regard to safety, the results refer to all patients randomized who received at least one dose of the trial medication.

\section{Morning and evening peak expiratory flow}

Compared to the last week of baseline, morning PEF (fig. 1) in the morning group increased by 8 and $3 \mathrm{~L} \cdot \mathrm{min}^{-1}$ after 4 and 8 weeks of treatment, respectively, which was not significant. In the evening group the improvements at the corresponding time points amounted to 24 and $30 \mathrm{~L} \cdot \mathrm{min}^{-1}(\mathrm{p}<0.005)$ (table 3$)$. The difference between treatments at 8 weeks was significant $(\mathrm{p}<0.05)$.

The change in evening PEF after 8 weeks of treatment was $7 \mathrm{~L} \cdot \mathrm{min}^{-1}$ in the morning group (NS versus baseline) and $16 \mathrm{~L} \cdot \mathrm{min}^{-1}(\mathrm{p}<0.05)$ in the evening group (table 3 ), resulting in a nonsignificant mean difference between treatments of $10 \mathrm{~L} \cdot \mathrm{min}^{-1}$.

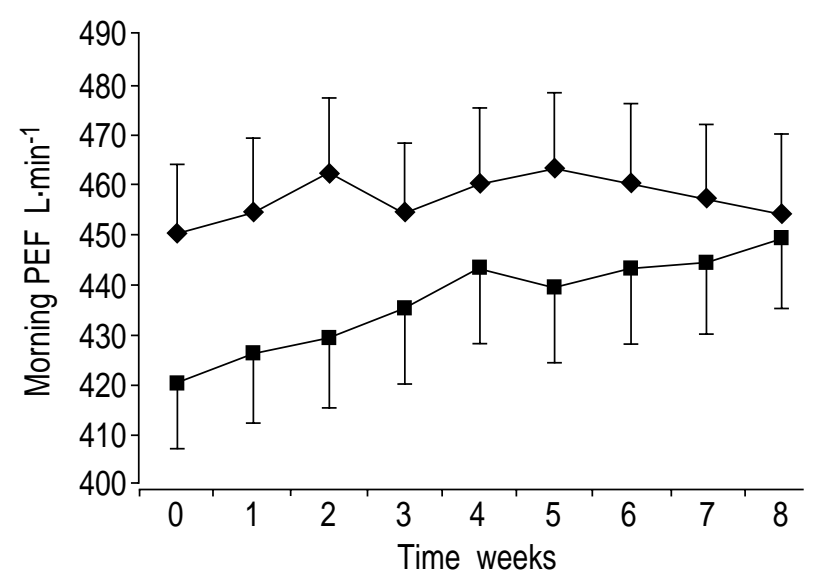

Fig. 1. - Time course mean morning peak expiratory flow (PEF); mean values and SEM. Time point " 0 " refers to the values recorded during the last week of the baseline period. $\downarrow$ : morning; : evening. 
Table 3. - Change in efficacy variables (end versus start of 8 weeks treatment)

\begin{tabular}{|c|c|c|c|}
\hline Variable & Morning & Evening & Morning - evening: \\
\hline FFEV1 L & $0.31(0.20-0.43)$ & $0.31(0.19-0.43)$ & $0.00(-0.15-0.15)$ \\
\hline FVC L & $0.19(0.06-0.33)$ & $0.22(0.08-0.36)$ & $-0.02(-0.19-0.14)$ \\
\hline Morning PEF $\mathrm{L} \cdot \mathrm{min}^{-1}$ & $3(-14-20)$ & $30(13-48)$ & $-27(-50--5)$ \\
\hline Evening $\mathrm{PEF} \mathrm{L} \cdot \mathrm{min}^{-1}$ & $7(-10-23)$ & $16(0.4-32)$ & $-10(-31-11)$ \\
\hline Total daily symptom score & $-0.38(-0.80--0.24)$ & $-0.50(-0.87--0.29)$ & $0.02(-0.29-0.43)$ \\
\hline Daily use rescue medication, puffs $\cdot 24 \mathrm{~h}^{-1}$ & $-0.36(-1.00--0.29)$ & $-0.36(-1.05--0.21)$ & $0(-0.43-0.43)$ \\
\hline
\end{tabular}

Lung function: least-squares means and $95 \%$ confidence intervals $(\mathrm{Cl})$; symptoms and rescue medication: within groups; median and 95\% CI, between groups: distribution-free point estimate and 95\%-CI. FEV1: Forced expiratory volume in one second; FVC: forced vital capacity; PEF: peak expiratory flow.

\section{Spirometry}

Compared to the last baseline reading, FEV1 and FVC increased significantly (at least $\mathrm{p}<0.05$ ) after 8 weeks of treatment in both treatment groups (table 3) and morning and evening administration proved to be equally effective. A similar improvement was observed after 4 weeks of treatment with increases amounting to 200 and $300 \mathrm{~mL}$ in the morning and evening for FEV1 and 150 and $250 \mathrm{~mL}$ for FVC, respectively. The time course of effect separated for patients either pretreated with inhaled steroids or not, is shown in figure 2 .

\section{Symptoms}

After both morning and evening administration of ciclesonide, total daily asthma symptoms improved significantly (at least $\mathrm{p}<0.001$ ) after 4 (data not shown) and 8 weeks of treatment (table 3 ). While during baseline only $21 \%$ and $24 \%$ of patients in the morning and evening group, respectively, were symptom-free, the percentage of patients without symptoms more than doubled (53\% and 55\%) after 8 weeks of treatment. No significant difference was found between groups for the total daily score as well as for symptoms reported during the day and night, respectively.

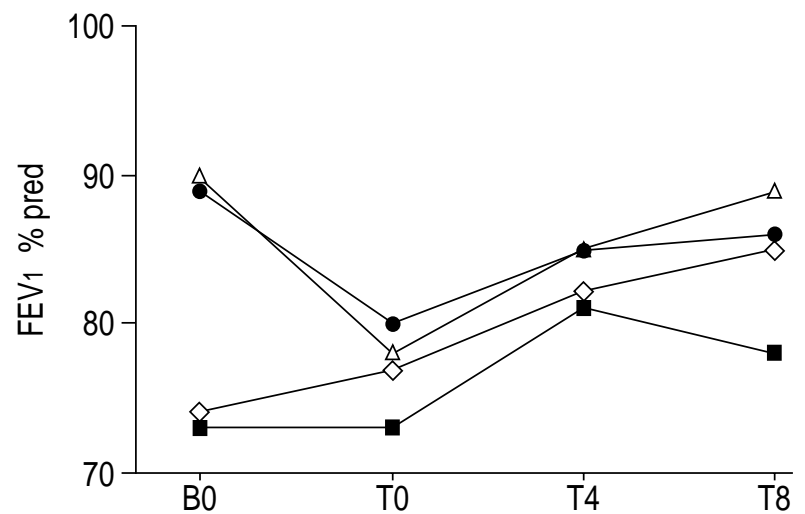

Fig. 2. - Time course in forced expiratory volume in one second $\left(F E V_{1}\right)$ in patients either with or without pretreatment with inhaled corticosteroids prior to the study. B0: start of baseline period; T0: time of randomization; T4/T8: after 4/8 weeks of treatment. $\diamond$ : morning, not pretreated; $\mathbf{\square}$ : evening, not pretreated; $\bullet$ : morning pretreated; $\triangle$ : evening pretreated.

\section{Use of rescue medication}

In both groups the use of rescue medication was significantly (at least $\mathrm{p}<0.05$ ) reduced after 4 (data not shown) and 8 weeks of treatment (table 3 ). The improvement was comparable in both groups and no significant difference between the groups was found. The same holds true for the percentage of rescue medication free days although this was slightly higher in the morning group (54\% versus $46 \%$ in the evening group).

\section{Lack of efficacy}

Lack of efficacy was described in the study protocol as asthma exacerbations to be treated with oral steroids. Eight out of 209 randomized patients $(3.8 \%)$ in total (four in each group) experienced lack of efficacy.

\section{Efficacy rating}

Both the investigators and patients rated ciclesonide as being "very effective" or "effective" in $61 \%$ and $63 \%$, respectively, of the patients treated in the morning, and in $71 \%$ and $63 \%$ with regard to the evening administration.

\section{Safety}

Overall, both treatment regimens were safe and well tolerated and there were no differences in the safety aspects between the groups. Eleven patients prematurely left the study because of adverse events; seven in the morning group and four in the evening group. This includes six patients who experienced lack of efficacy as described earlier. The other events leading to withdrawal were one case each of bronchial hypersecretion, nausea/vomiting, dyspnoea and panic attacks, the latter occurring under placebo.

The most frequent adverse events $(\geqslant 3 \%)$ concerned the respiratory system (upper respiratory infection: $6.7 \%$; asthma: $6.2 \%$; bronchitis: $4.3 \%$ ). Two patients $(1 \%)$ reported voice alterations; no case of candidiasis was described.

Mean 24-h urinary cortisol excretion normalized for creatinine amounted to 8.49 and $8.10 \mathrm{nmol} \cdot \mathrm{mmol}$ 
creatinine $^{-1}$ (morning and evening group, respectively) during baseline. At the end of treatment, the respective values were 10.47 and $13.88 \mathrm{nmol} \cdot \mathrm{mmol}$ creatinine $^{-1}$. Mean differences within treatment groups (morning: $1.98 \mathrm{nmol} \cdot \mathrm{mmol}$ creatinine $^{-1}(95 \%$ confidence interval (CI): $-2.34-6.33)$; evening: $5.78 \mathrm{nmol} \cdot \mathrm{mmol}$ creatinine $^{-1}(95 \%$ CI: $\left.-6.51-18.06)\right)$ were not significant as were the differences between treatment groups (-3.80 nmol-mmol creatinine ${ }^{-1}$ (95\% CI: -16.79-9.19)). Values below the normal range occurred in 23 patients at baseline and in 15 at the end of the treatment in the morning group, and in 28 and 24 patients, respectively, in the evening group.

\section{Discussion}

Results of the present study suggest that $200 \mu \mathrm{g}$ ciclesonide given once daily is effective in the treatment of mild-to-moderate asthma as assessed by lung function, symptoms, use of rescue medication and number of asthma exacerbations. Although the trial did not have a placebo or active control group, the consistent improvements above baseline values were not only statistically significant but also clinically relevant, and thus support this conclusion.

Morning and evening administration was shown to be equally effective for the different variables, except for morning PEF where the improvements after evening dosing were more prominent. When looking at individual patient responses (see 95\% CI in table 3) obviously some patients deteriorated while others improved resulting in no relevant change overall. Hence, the response pattern to a dose given in the morning seems to be more heterogeneous compared to evening administration where all patients had higher values at the end of treatment referred to baseline. It should be noted that baseline morning PEF values in the morning group were higher than in the evening group. It, therefore, might be suspected that a ceiling effect occurred and that the morning group had in fact no room for further improvement. This speculation is, however, not supported by the covariance analysis, which took the differences in baseline into account and nevertheless suggested a significant difference in outcome for the two groups. As two other trials with ciclesonide showed that 100 and $200 \mu \mathrm{g} \cdot \mathrm{day}^{-1}$ given in the morning significantly improved morning PEF compared to placebo, no explanation is, therefore, readily available why this variable was unchanged in the morning group of the current study.

When selecting the optimal time point of administration for an individual patient, the following aspects may be relevant: patient compliance, the fact that asthma can have an important nocturnal component and safety considerations. With regard to compliance, morning dosing of inhaled steroids is favoured [13]. Concerning safety, pharmacokinetic/pharmacodynamic modelling suggests that inhalation of steroids in the afternoon might cause the least cortisol suppression, with the optimum time point being determined by the terminal elimination half-life of the respective drug: i.e. an administration time point of 15:00 h was proposed for fluticasone propionate and of 19:00 h for flunisolide [14]. Finally, due to the circadian rhythm in lung function, hyperresponsiveness, circulating cells and mediators in asthma, it might be expected that based on the results with oral and intravenous steroids [15, 16], afternoon/evening dosing of inhaled steroids is preferable to morning dosing for alleviation of nocturnal worsening in asthma.

Morning administration of budesonide was shown to be superior to placebo [17] and the authors concluded that recommendation for the time of dosing may well be left to the individual physician. Several studies evaluated the efficacy of evening dosing of budesonide and with the exception of one trial [4] they all found that evening administration is at least as effective as twice-daily dosing [18-20]. In addition, JONEs et al. [8] addressed the issue of time of dosing and concluded that morning and evening administration of budesonide are equi-effective. Similar findings were made for flunisolide; although for morning PEF and daytime use of rescue medication there was a trend in favour of evening dosing [21]. The efficacy of $100 \mu \mathrm{g}$ fluticasone propionate twice-daily was generally greater than a $200 \mu \mathrm{g}$ once-daily morning regimen in patients pretreated with inhaled steroids, whereas no such difference was seen in patients pretreated with bronchodilators only [22]. Hence, there is clear evidence from the literature that once-daily dosing of inhaled steroids is an effective regimen for the treatment of mild-to-moderate asthma. No final conclusion is yet possible about the ideal time point of administration although some studies suggest that morning dosing might be less efficacious. In fact PINCUS and coworkers [13, 23] proposed that administration of inhaled steroids in the afternoon between 15:00-17:30 h might result in the most pronounced asthma control. However, afternoon administration does not necessarily increase compliance and as the present trial suggests, relevant improvement can also be achieved by evening dosing between 16:00-20:00 h, the time window for optimal administration of inhaled steroids might in fact be wider. This is also in line with a study investigating the efficacy of a single dose of $1,000 \mu \mathrm{g} \mathrm{BDP} \cdot \mathrm{day}^{-1}$ in the late afternoon $(17: 00 \mathrm{~h})$ and at bedtime $(22: 00 \mathrm{~h})$ showing comparable asthma control for both regimens [24].

The safety data of the current trial suggest that ciclesonide was well tolerated. No influence on HPA axis was found as judged by 24-h urinary cortisol suppression. This is in line with the results of a study in healthy volunteers where the 24-h mesor for serum cortisol under ciclesonide $(800 \mu \mathrm{g})$, given either in the morning or in the evening for one week, was $2-6 \%$ lower compared to placebo indicating that ciclesonide lacks relevant systemic effects [25]. Hence, from a safety point of view ciclesonide can be administered both in the morning or evening.

Although the question on the ideal time point of once-daily administration of inhaled steroids warrants further investigation, the current study suggests that ciclesonide can be given either in the morning or in the evening so that patient preference and individual medical needs can be addressed. 
Acknowledgements. The following additional investigators participated in the morning or evening (MORE) trial: Belgium: J. Bruart, La Louviere; K.P.J. De Schepper, St. Niklaas; J-B. Martinot, Namur; K.R. Van Oortegem, Kortrijk; A.F.J. Verstraeten, Gent; J-P. d'Odémont, Mons. France: H. Audouin, Nantes; Y. Bassegoda, Thionville; P. Bihet, Saint-Brieuc; J-C. Farouz, Bordeaux; M. Grosclaude, Guilherand-Granges; P. Hyvernat, Lyon; J.P. Moreau, Rennes; I. Petit-Levy, Montauban; C. Prenat, Besancon; J-Y. Rigault, Lorient; J-F. Vidal, Montelimar; J-M. Zordan, Hagondage. The Netherlands: A.J.M. Bax, Bilthoven; H.C.C. Christiaanse-Vandewalle, Rotterdam; A.C. Coenders, Amsterdam; J.P.H.M. Creemers, Eindhoven; P. De Graaf, Utrecht; P.F.L.H.M. Deckers, Rotterdam; A.P.M. Greefhorst, Hengelo; A.P. Sips, Utrecht; J.A. van Noord, Heerlen; UK: M.E. Johnson, Barnsley; R. Kneen, Saltash; A. Middleton, Fowey.

\section{References}

1. Rand CS. Patient and regimen-related factors that influence compliance with asthma therapy. Eur Respir Rev 1998; 8: 270-274.

2. Toogood JH, Baskerville JC, Jennings B, Lefcoe NM, Johansson SA. Influence of dosing frequency and schedule on the response of chronic asthmatics to the aerosol steroid budesonide. J Allergy Clin Immunol 1982; 70: 288 - 298.

3. Reed CE. Aerosol glucocorticoid treatment of asthma: adults. Am Rev Respir Dis 1990; 141: S82-S88.

4. Weiner P, Weiner M, Azgad Y. Long term clinical comparison of single versus twice daily administration of inhaled budesonide in moderate asthma. Thorax 1995; 50: $1270-1273$.

5. Campbell LM. Once-daily inhaled corticosteroids in mild to moderate asthma. Improving acceptance of treatment. Drugs 1999; 58: Suppl. 4. 25-33.

6. Taylor DA, Jensen MW, Kanabar V, et al. A dosedependent effect of the novel inhaled corticosteroid ciclesonide on airway responsiveness to adenosine- $5^{\prime}$ monophosphate in asthmatic patients. Am J Respir Crit Care Med 1999; 160: 237-243.

7. Dahl R, Nielsen LP, Christensen MB, Engelstätter R. Ciclesonide - an inhaled corticosteroid prodruginhibits allergen induced early and late phase reactions. Eur Respir J 1998; 12: Suppl. 28, 62.

8. Jones AH, Langdon CG, Lee PS, et al. Pulmicort $\mathbb{R}$ Turbohaler $(\mathrm{R}$ once daily as initial prophylactic therapy of asthma. Respir Med 1994; 88: 293-299.

9. American Thoracic Society. Standards for the diagnosis and care of patients with chronic obstructive pulmonary disease (COPD) and asthma. Am Rev Respir Dis 1987; 136: 225-244.

10. Quanjer PH, Dalhuijsen A, van Zomeren BC. Summary equations of reference values. Bull Eur Physiopathol Respir 1993; 19: Suppl. 5, 45-51.

11. Davies RJ, Stampone P, O'Connor BJ. Hydrofluoroalkane-134a beclomethasone dipropionate extrafine aerosol provides equivalent asthma control to chlorofluorocarbon beclomethasone dipropionate at approximately half the total daily dose. Respir Med 1998; 92: Suppl. A, 23-31.

12. International conference on harmonisation of technical requirements for registration of pharmaceuticals for human use. ICH harmonised tripartitate guideline: statistical principles for clinical trials. Statist Med 1999; 18: 1905-1942.

13. Pincus DJ, Humeston TR, Martin RJ. Further studies on the chronotherapy of asthma with inhaled steroids: the effect of dosage timing on drug efficacy. J Allergy Clin Immunol 1997; 100: 771 - 774.

14. Maibohm B, Hochhaus G, Rohatagi S, et al. Dependency of cortisol suppression on the administration time of inhaled corticosteroids. J Clin Pharmacol 1997; 37: 704-710.

15. Beam WR, Ballard RD, Martin RJ. Spectrum of corticoid sensitivity in nocturnal asthma. Am Rev Respir Dis 1992; 145: $1082-1086$.

16. Beam WR, Weiner DE, Martin RJ. Timing of prednisolone and alterations of airway inflammation in nocturnal asthma. Am Rev Respir Dis 1992; 146: $1524-1530$.

17. McFadden ER, Casale TB, Edwards TB, et al. Administration of budesonide once daily by means of Turbuhaler to subjects with stable asthma. J Allergy Clin Immunol 1999; 104: 46-52.

18. Campbell LM, Gunn SD, Sweeney D, et al. Once daily budesonide: effective control of moderately severe asthma with $800 \mathrm{mcg}$ once daily inhaled via Turbohaler when compared with $400 \mu \mathrm{g}$ twice daily. Eur J Clin Res 1995; 7: 1-14.

19. Venables TL, Addlestone MB, Smithers AJ, et al. A comparison of the efficacy and patient acceptability of 20 once daily budesonide via Turbohaler and twice daily fluticasone propionate via disk-inhaler in an equal daily dose of $400 \mu \mathrm{g}$ in adult asthmatics. $\mathrm{Br}$ $J$ Clin Res 1996; 7: 15-32.

20. Campbell LM, Bodalia B, Gogbashian CA, Gunn SD, Humphreys PJ, Powell JP. Once-daily budesonide: $400 \mu \mathrm{g}$ once daily is as effective as $200 \mu \mathrm{g}$ twice daily in controlling childhood asthma. Int J Clin Pract 1998; 52: $213-219$.

21. ZuWallack RL, Rosen JP, Cohen L, et al. The effectiveness of once-daily dosing of inhaled flunisolide in maintaining asthma control. J Allergy Clin Immunol 1997; 99: $278-285$.

22. Wolfe J, Rooklin A, Grady J, et al. Comparison of once- and twice-daily dosing of fluticasone propionate 200 micrograms per day administered by Diskus device in patients with asthma treated with or without inhaled corticosteroids. J Allergy Clin Immunol 2000; 105: $1153-1161$.

23. Pincus DJ, Szefler SJ, Ackerson LM, Martin RJ. Chronotherapy of asthma with inhaled steroids: the effect of dosage timing on drug efficacy. J Allergy Clin Immunol 1995; 95: $1172-1178$.

24. Gagnon M, Côte J, Milot J, Turcotte H, Boulet L-P. Comparative safety and efficacy of single or twice daily administration of inhaled beclomethasone in moderate asthma. Chest 1994; 105: 1732 - 1737.

25. Weinbrenner A, Hüneke $\mathrm{D}$, Zschiesche $\mathrm{M}$, et al. Circadian rhythm of serum cortisol after repeated inhalation of the new topical steroid ciclesonide. Eur J Clin Pharmacol 1998; 54: A27. 